\title{
Designing a Biosensor Using a Photonic Quasi-Crystal Fiber with Fan-shaped Analyte Channel
}

\author{
Suoda Chu ${ }^{1}$, K. Nakkeeran ${ }^{1}$, G. Melwin ${ }^{2}$, Sumeet S. Aphale ${ }^{1}$, P. Ramesh \\ $\mathrm{Babu}^{2}$ and K. Senthilnathan ${ }^{2}$ \\ 1 School of Engineering,Fraser Noble Building, University of Aberdeen, Aberdeen \\ AB24 3UE, UK \\ 2 Department of Physics, School of Advanced Sciences, VIT University, Vellore-632 \\ 014, Tamil Nadu, India
}

$<\mathrm{k}$.nakkeeran@abdn.ac.uk>

\begin{abstract}
In this research work, we design a biosensor using a six-fold photonic quasi-crystal fiber with a fan-shaped analyte channel based on surface plasmon resonance (SPR). We numerically analyze both the dispersion relations and loss spectra for three different refractive indices of the analyte, $n_{a}$, using finite element method. Through optimization of the structure, we find that the proposed biosensor exhibits a maximum refractive index sensitivity of $3200 \mathrm{~nm} / \mathrm{RIU}$ and a resolution of $3.12 \times 10^{-5} \mathrm{RIU}$ when $n_{a}$ is increased from 1.41 to 1.43 . We infer that the coupling between the core mode and SPR mode can be explained as a complete coupling of the loss matching condition or an incomplete coupling of the phase matching condition. Owing to the ease of fabrication of the proposed biosensor with an average sensitivity of $2250 \mathrm{~nm} / \mathrm{RIU}$, we envisage that this biosensor could turn out to be a versatile instrument for detecting the biomolecules.
\end{abstract}

Keywords: Fan-shaped, Photonic Quasi-crystal fiber, Refractive index sensor, Surface Plasmon Resonance, Sensitivity.

\section{Introduction}

As a surface plasmon resonance (SPR) based senor, photonic biosensors with remarkable advantages such as extremely high sensitivity, fast response, labelfree, capability of removable detection, online sensing, etc give rise to a robust and effective solution for direct sensing application [1]. It is widely studied by a number of researchers in various fields like medical diagnostic, bio-chemical detection and organic chemical detection for the investigation of bio-molecular interaction and the recognition of target analytes [2].

It is well known that SPR is essentially an electromagnetic mode, due to the collective resonant oscillation of free electrons, which is stimulated by incident light at the interface of metal and dielectric material [3]. At a specific wavelength, the coupling will fulfill with the phase matching condition which can be explained 
by the propagation constants equivalence of a core-guided mode and a plasmonic mode. The first SPR experiment work for bio-sensing and gas detection was presented by Liedberg et al. in 1983 [2]. Conventionally, the SPR biosensors widely available in the market work with the Krechmann configuration which is based on prism-coupling theory. The sensing ability of this kind of prism based SPR sensors is quite good enough to detect even small variation in the refractive index of analyte at the prism-metal interface [2][3]. However, due to their bulk size and complicated design and structure, it is not easy to realize a portable sensor [4][5].

The availability of optical fiber SPR sensor has made a break through in this limitation because of its compact small size and design flexibility. The first optical fiber SPR sensor with a maximum refractive index measurement resolution of $7.5 \times 10^{-4} \mathrm{RIU}$ at $900 \mathrm{~nm}$ was proposed by Jorgenson in 1993 [6]. Further, multi-mode fibers were replaced by single mode fibers for higher sensitivity and sharper loss peak [5]. With the development on photonic crystal fibers (PCFs), it is possible to control the optical characteristics of PCF by having different structures and by varying the structural parameters. The sensing ability and sensing range witnessed a further improvement with the optimization of structure [2]. Later on, a lot of PCF-SPR biosensors with different structures such as, multi-core holey fiber in 2012 [1], large size microfluidic channel PCF in 2012 [7], square lattice PCF in 2014 [8], D-shaped PCF in 2015 [9] have been proposed for enhancing the sensitivity. Due to the limitations in the fabrication technology, currently it is quite difficult to coat the metal part onto the air holes. Among these novel designs, the D-shaped PCF was considered as a most promising way to fabricate by side-polishing technology [10]. Besides the common advantages, the D-shaped PCF-SPR biosensor can easily be coated with the metal layer outside the PCF air holes.

Recently, Aruna Gandhi et al proposed a refractive index based biosensor using a photonic quasi-crystal fiber (PQF) [11]. This PQF is a micro-structured fiber with quasi periodicity that has a long-range order with aperiodic arrangement in the cladding region [12]. Based on our previous work on a six-fold PQF biosensor [4], in this work, we propose a six-fold PQF biosensor where a fanshaped part of the cladding is removed. Here, the cladding material is made of poly methyl methacrylate (PMMA). The structure proposed in this paper is based on the D-shaped fiber. Then, a sector is removed as the resonant wavelength is found to be exceeding the wavelength range for defining the refractive index of PMMA. For a D-shaped PQF biosensor, the matching resonance wavelength is found to be $1.6 \mu \mathrm{m}$ when the refractive index of analyte is 1.40 . The matching resonance wavelength gets increased as the analyte refractive index is increased. As the maximum wavelength used in PMMA refractive index equation is limited to $1.6 \mu \mathrm{m}$, for a higher analyte refractive index, say, 1.42, the matching wavelength must be more than the maximum value of the wavelength range. Furthermore, the coupling phenomenon in this SPR sensing system occurs either because of the incomplete coupling of the phase matching condition or the complete coupling of the loss matching condition. This plays a crucial role 
during the resonant process between the fundamental mode and SPR mode [1]. The paper is laid out as follows. We discuss, a detailed geometrical structure and theoretical modeling in section 2. Section 3 deals with the results and analysis of the proposed work. Finally, we conclude the work in section 4 .

\section{Simulated geometrical structure and theoretical modeling}

The structure of this designed SPR biosensor is shown in Fig. 1. Unlike the common D-shaped PCF structure, a fan-shaped sector with the central angle of $72^{\circ}$ and $6.2 \mu \mathrm{m}$ in depth is removed from the original circular structure. It is because that the gold layer can be easily coated at the interface between analyte and PMMA cladding. This empty space can be used to fill in with the analyte liquid as a sensor channel. The inter air holes are arranged in a fixed distance of pitch, $\Lambda$, of $2 \mu \mathrm{m}$. The diameter of all the air holes is kept as $1 \mu \mathrm{m}$. The gold layer thickness is fixed as $50 \mathrm{~nm}$. In order to investigate the mode coupling of this sensor, the finite element method (FEM) is used for simulation and a perfectly matched layer (PML) boundary condition is applied for absorbing the radiation power.

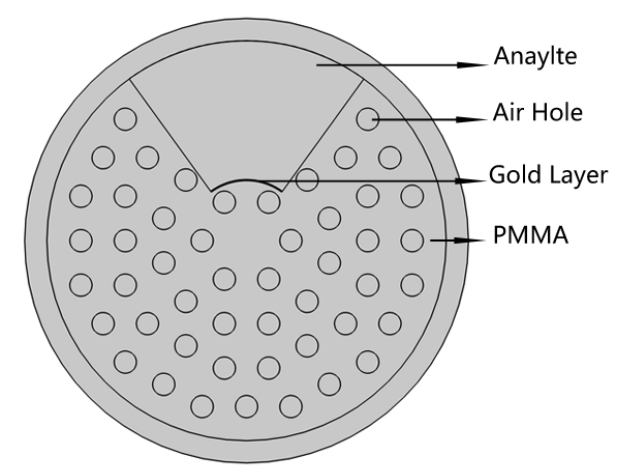

Fig. 1. Cross-section of the prposed six-fold PQF biosensor.

The background material of this sensor is PMMA. For an accurate calculation, the wavelength dependence of the refractive index of PMMA is defined by the following equation which was reported by Beadie [13]:

$$
n^{2}(\lambda)=a_{0}+a_{1} \lambda^{2}+a_{2} \lambda^{4}+a_{3} \lambda^{-2}+a_{4} \lambda^{-4}+a_{5} \lambda^{-6}+a_{6} \lambda^{-8},
$$

where $a_{0}=2.1778, a_{1}=6.1209 \mathrm{e}-3, a_{2}=-1.5004 \mathrm{e}-3, a_{3}=2.3678 \mathrm{e}-2, a_{4}=-4.2137 \mathrm{e}-$ $3, a_{5}=7.3417 \mathrm{e}-4$ and $a_{6}=-4.5042 \mathrm{e}-5$. Here, $\lambda$ represents the incident light wavelength in vacuum. It should be noted that the fit coefficients are valid and can be used in the calculation of refractive index of PMMA when the temperature 
condition is around $20.1 \mathrm{C}$ with an experimentally verified incident wavelength ranging between $0.4 \mu \mathrm{m}$ and $1.6 \mu \mathrm{m}$. Next, the dielectric constant of gold is characterized by the Drude model [14]. The fan-shaped channel is filled with the analyte liquid sample with the refractive index changing from 1.41 to 1.43 .

\section{Results and Analysis}

Figures 2(a) and 2(b) illustrate both the distribution of electric field of a fundamental mode and a plasmonic mode of the fan-shaped channel PQF biosensor at $1100 \mathrm{~nm}$. It can be seen clearly from Fig. 2(a) that the power of core-guided mode is almost concentrated in the core area while there is negligible plasmonic mode power existing at the interface between the PMMA and the fan-shaped analyte channel. This indicates that there is no power exchange between those two modes and hence, there is no coupling taking place at this wavelength.

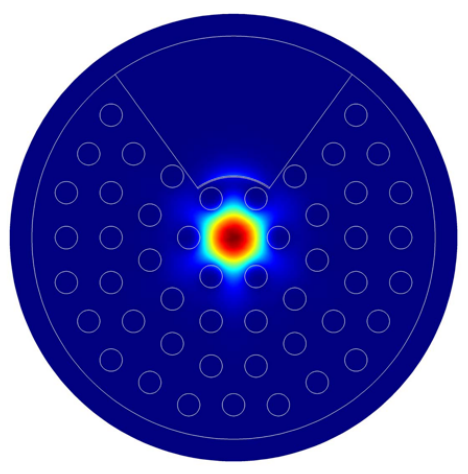

(a)

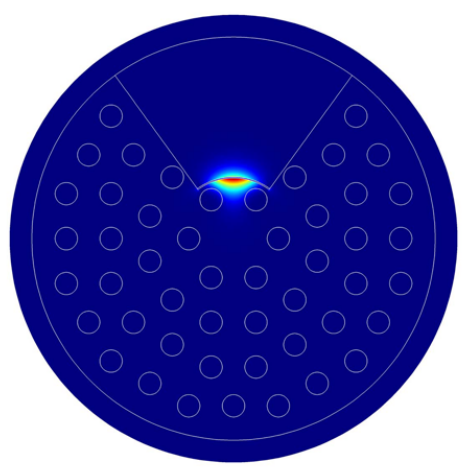

(b)

Fig. 2. (a) The distribution of electric field of fundamental mode and (b) the distribution of electric field of plasmonic mode at $1100 \mathrm{~nm}$ when $n_{a}=1.43$. 
In any plasmonic sensor, it is known that the plasmonic mode arises as a consequence of the enrgy transfer from the fundamental mode. Figs. 3(a) and 3 (b) represent the electric field distribution of the fundamental mode and plasmonic mode at a mode matching wavelength of $1390 \mathrm{~nm}$. As against the case of Fig. 2(a) and 2(b), one can visualize the power transfer from the fundamental mode as plasmonic which reflects the peak in the loss spectrum. The leaky core mode energy excites the surface plasmon waves (SPWs) at the interface of gold layer. Moreover, it has a maximum loss at this particular wavelength so that we can make use of this confinement loss peak for sensing. Here, the confinement loss $\alpha$ is calculated by [15],

$$
\alpha_{\text {loss }}=\frac{40 \pi}{\lambda \ln 10} \operatorname{Im}\left(n_{n e f f}\right)
$$

where $\lambda$ is the wavelength of incident light in vacuum and $\operatorname{Im}\left(n_{n e f f}\right)$ represents the imaginary part of effective refractive index of the core-guided mode.

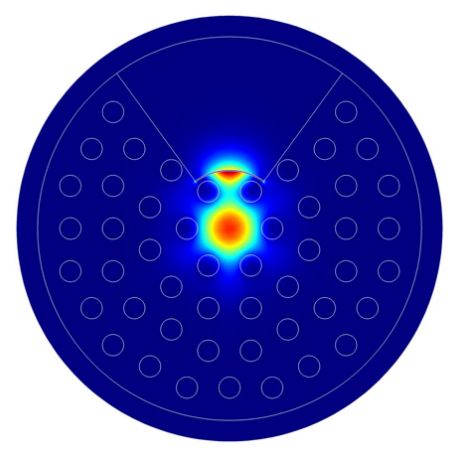

(a)

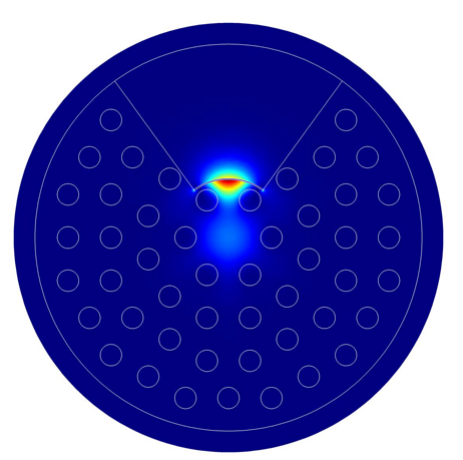

(b)

Fig. 3. (a) The distribution of electric field of fundamental mode and (b) the distribution of electric field of plasmonic mode at $1390 \mathrm{~nm}$ when $n_{a}=1.43$. 


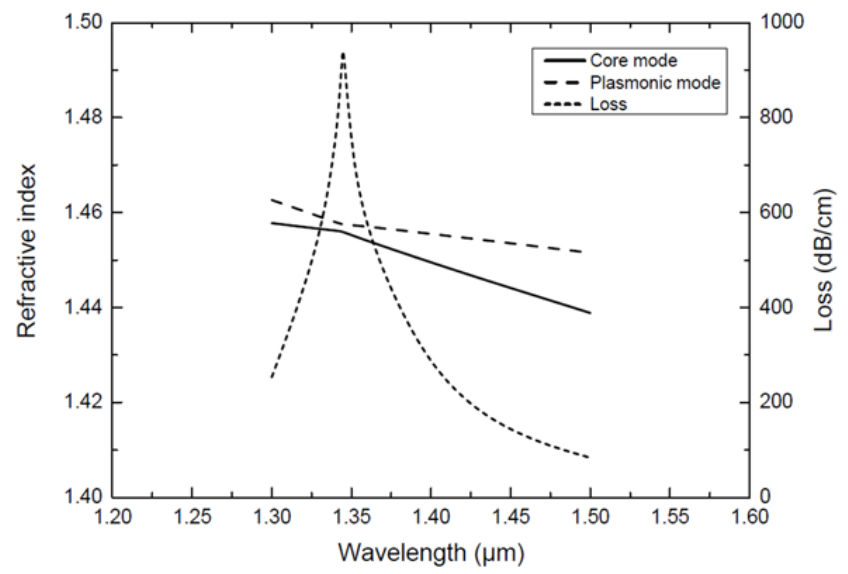

(a)

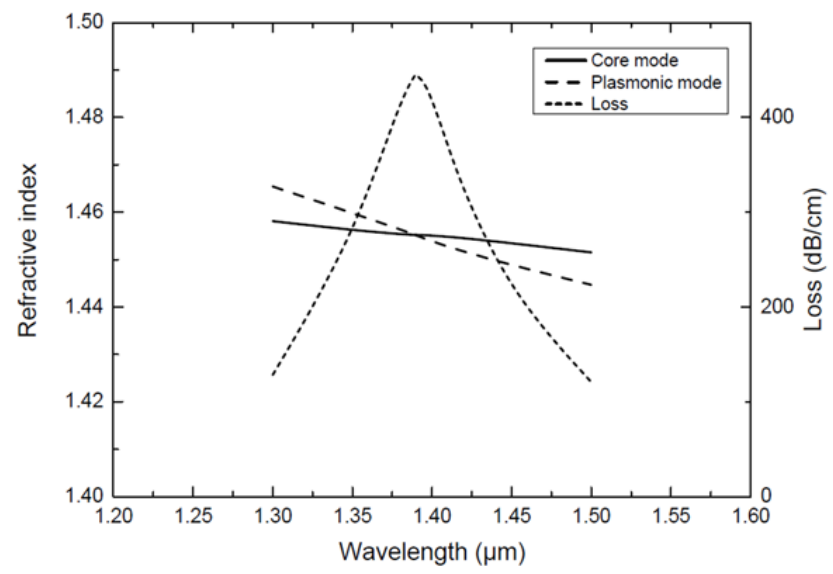

(b)

Fig. 4. (a) Dispersion relations of the fundamental mode and SPR mode and confinement loss when $n_{a}=1.41$. (b) Dispersion relations of the fundamental mode and SPR mode and confinement loss when $n_{a}=1.43$.

Figures 4(a) and 4(b) show the dispersion relation of the fundamental mode and plasmonic mode along with the fundamental mode loss when the analyte refractive indices are 1.41 (Fig. 4(a)) and 1.43 (Fig. 4(b)), respectively. It is obvious that, at resonance wavelength, a maximum power loss is reached due to the maximum leakage of fundamental mode. Comparing the real part of effec- 
tive refractive indices of fundamental mode and SPR mode, there is a crossing between the modes in Fig. 4(b). However, such a crossing is not observed in Fig. 4(a). The real parts of $n_{\text {eff }}$ of the fundamental mode and the SPR mode for the increasing values of the analyte refractive index are getting closer to each other. However, for further increase in coupling wavelength they get crossed with a intrsection point. This can be explained by the coupled-mode theory [1] and [3]. In Fig. 4(a), where the imaginary parts are equal to each other, a complete coupling does occur. On the other hand, from the results of Fig. 4(b), when the real parts of effective refractive indices of the two modes are equal, an incomplete coupling occurs [16]. From the detailed numerical results, it is observed that the biosensor works under the complete coupling of the loss matching condition when the analyte refractive index is equal to 1.41. Conversely, there is an incomplete coupling of the phase matching condition when the analyte refractive index is above 1.41. This finding indicates that the SPR phenomenon for this biosensor exhibits a complete coupling with lower analyte refractive indices and then turns into incomplete coupling with the increasing analyte refractive index values.

Figure 5 indicates loss spectra when $n_{a}$ is increased from 1.41 to 1.43. From the result, it is clear that the loss peak is getting shifted towards the longer wavelength side with the periodical decrement in the loss as and when the refractive of the analyte is increased from 1.41 to 1.43 . Based on the sensitivity calculation of the proposed biosensor, for a $0.1 \mathrm{~nm}$ peak-wavelength resolution instrument, the maximum sensitivity is $3200 \mathrm{~nm} / \mathrm{RIU}$ and the average sensitivity is 2250 $\mathrm{nm} / \mathrm{RIU}$.

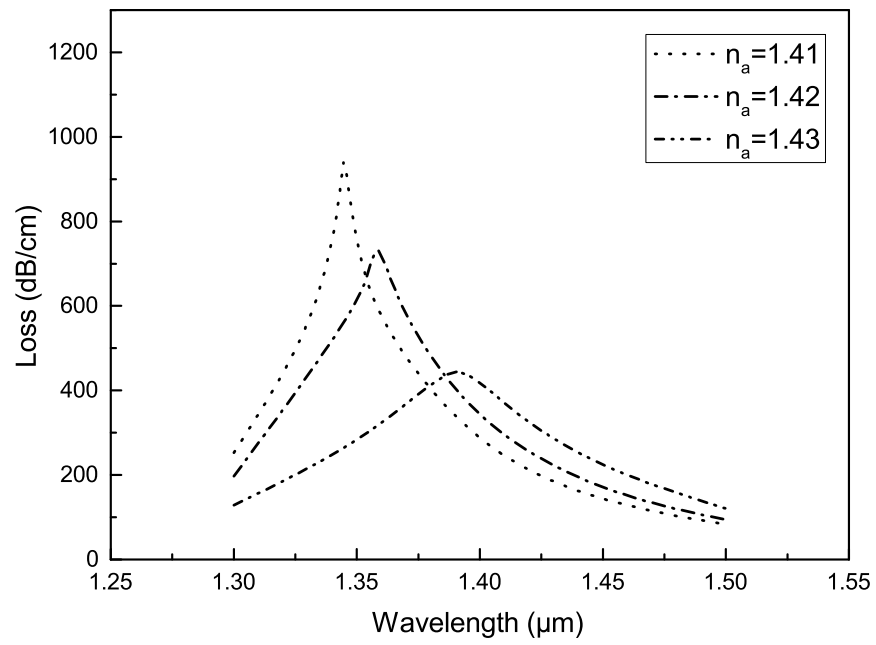

Fig. 5. The loss spectra as a function of wavelength when the refractive index of analyte is varied from 1.41 to 1.43 . 


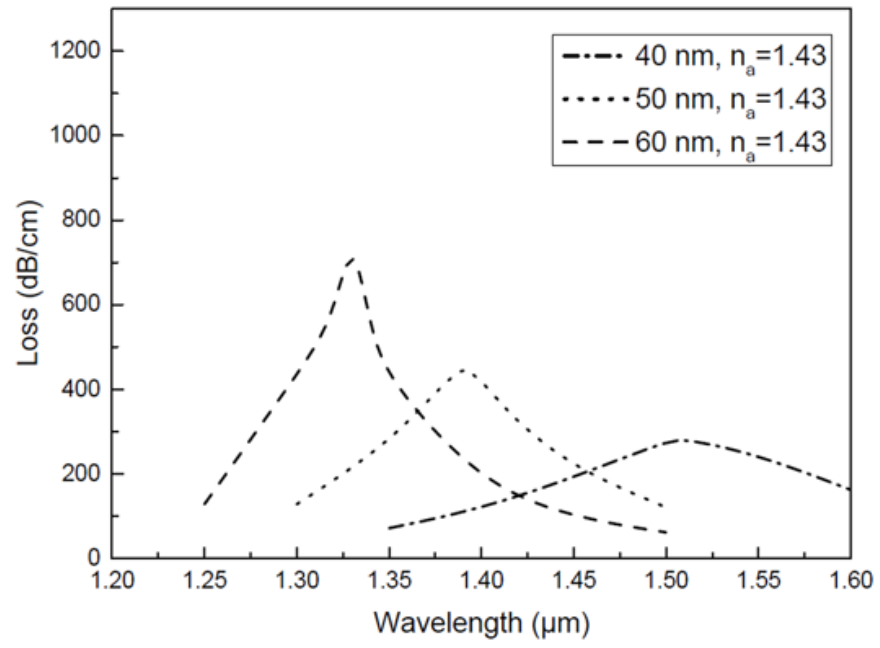

Fig. 6. The loss spectra as a function of wavelength with different thickness of gold layer varying from 40 to $60 \mathrm{~nm}$.

It is well known that the gold layer thickness is a significant parameter in determining the half-width and depth of the resonance peak [17]. Therefore, the impact of different gold layer thickness has also been studied. Figure 6 shows the simulated results with the gold layer thickness of $40 \mathrm{~nm}, 50 \mathrm{~nm}$ and $60 \mathrm{~nm}$ which indicate that the loss peak decreases and shifts to the longer wavelength side as the gold layer thickness increases.

\section{Conclusion}

We have proposed a six-fold photonic quasi-crystal fiber biosensor with a fanshaped analyte channel in this paper. Due to its unique structure, it exhibits two different kinds of coupling conditions, namely, the complete coupling and incomplete coupling. Both these couplings could produce the surface plasmon resonance phenomenon. A maximum refractive index sensitivity of $3200 \mathrm{~nm} / \mathrm{RIU}$ and a resolution of $3.12 \times 10^{-5}$ RIU have been achieved for a sensing range from 1.41 to 1.43 . It is worthwhile to emphasize that the gold layer coating process in the fan-shaped cavity is easier than coating into the air holes. We are of the opinion that with the existing state of the art of the fabrication technologies, the realization of the proposed biosensor would be a viable one. 


\section{References}

1. Binbin Shuai, Li Xia, Yating Zhang, and Deming Liu. A multi-core holey fiber based plasmonic sensor with large detection range and high linearity. Opt. Express, 20(6):5974-5986, Mar 2012.

2. Ahmmed A. Rifat, G. Amouzad Mahdiraji, Desmond M. Chow, Yu Gang Shee, Rajib Ahmed, and Faisal Rafiq Mahamd Adikan. Photonic crystal fiber-based surface plasmon resonance sensor with selective analyte channels and graphenesilver deposited core. Sensors, 15(5):11499-11510, 2015.

3. Guowen An, Shuguang Li, Xin Yan, Xuenan Zhang, Zhenyu Yuan, Haiyang Wang, Yanan Zhang, Xiaopeng Hao, Yaonan Shao, and Zhicong Han. Extra-broad photonic crystal fiber refractive index sensor based on surface plasmon resonance. Plasmonics, 12(2):465-471, 2017.

4. Design a Biosensor Using a Stampli Photonic Quasi-Crystal Fiber. ACTA Press, 2016.

5. N. Luan and J. Yao. Surface plasmon resonance sensor based on exposed-core microstructured optical fiber placed with a silver wire. IEEE Photonics Journal, 8(1):1-8, Feb 2016.

6. Photonic crystal fiber based surface plasmon resonance chemical sensors. Sensors and Actuators B: Chemical, 202:557 - 567, 2014.

7. Pibin Bing, Jianquan Yao, Ying Lu, and Zhongyang Li. A surface-plasmonresonance sensor based on photonic-crystal-fiber with large size microfluidic channels. Opt. Appl, 42(3):493-501, 2012.

8. M. A. A. Mamun, M. A. Islam, and M. S. Alam. A square lattice photonic crystal fiber based surface plasmon resonance sensor with high sensitivity. In 2014 International Conference on Electrical Engineering and Information Communication Technology, pages 1-5, April 2014.

9. Wu Jing Shi Wei-Hua, You Cheng-Jie. D-shaped photonic crystal fiber refractive index and temperature sensor based on surface plasmon resonance and directional coupling. Acta Physica Sinica, 64(22):224221, 2015.

10. Yu Ying, Guang yuan Si, Fang jun Luan, Ke Xu, Yuan wei Qi, and Hong nan Li. Recent research progress of optical fiber sensors based on d-shaped structure. Optics and Laser Technology, 90:149 - 157, 2017.

11. M. S. Aruna Gandhi, S. Sivabalan, P. Ramesh Babu, and K. Senthilnathan. Designing a biosensor using a photonic quasi-crystal fiber. IEEE Sensors Journal, 16(8):2425-2430, April 2016.

12. S. Sivabalan and J. P. Raina. High normal dispersion and large mode area photonic quasi-crystal fiber stretcher. IEEE Photonics Technology Letters, 23(16):11391141, Aug 2011.

13. G. Beadie, Michael Brindza, Richard A. Flynn, A. Rosenberg, and James S. Shirk. Refractive index measurements of poly(methyl methacrylate) (pmma) from $0.4 \mu \mathrm{m}$ 1.6 um. Appl. Opt., 54(31):F139-F143, Nov 2015.

14. Alireza Hassani and Maksim Skorobogatiy. Design criteria for microstructuredoptical-fiber-based surface-plasmon-resonance sensors. J. Opt. Soc. Am. B, 24(6):1423-1429, Jun 2007.

15. Pei-pei Zhang, Jian-quan Yao, Hai-xia Cui, and Ying Lu. A surface plasmon resonance sensor based on a multi-core photonic crystal fiber. Optoelectronics Letters, 9(5):342-345, 2013.

16. Zhenkai Fan, Shuguang Li, Qiang Liu, Guowen An, Hailiang Chen, Jianshe Li, Dou Chao, Hui Li, Jianchen Zi, and Wenlong Tian. High sensitivity of refractive 
index sensor based on analyte-filled photonic crystal fiber with surface plasmon resonance. IEEE Photonics Journal, 7(3):1-9, 2015.

17. R. Otupiri, E. K. Akowuah, S. Haxha, H. Ademgil, F. AbdelMalek, and A. Aggoun. A novel birefrigent photonic crystal fiber surface plasmon resonance biosensor. IEEE Photonics Journal, 6(4):1-11, Aug 2014. 